\title{
QUINTA MESA REDONDA
}

1. Al doctor Báez: - ¿Qué conducta se debe observar con respecto a los embarazos posteriores de una madre que ha dado a luz con resultado positivo al tratamiento sico-profiláctico? ¿Necesita nuevo tratamiento?

Doctor Báez: - Nosotros tenemos madres que ya han dado a luz con preparación sico-profiláctica hasta cuatro veces, repitiendo la preparación consecutivamente; pero en estos casos tenemos pensado publicar, precisamente hemos visto que la causa fundamental por la cual la madre volvía al tratamiento era el temor. Así, comprobamos que la primera clase es indispensable; las otras, de anatomía y lo demás son mucho más fáciles de recordar o al menos de tenerlas presentes. Donde volvimos a insistir con mucha preocupación es en la relajación, la respiración, y sobre todo los ejercicios para el período expulsivo.

2. Del doctor Bussamara al doctor Báez: -Teniendo en cuenta que la sico-profilaxia tranquiliza, ayudando a la paciente a retardar su internación al hospital para el trabajo del parto, ¿qué se piensa en cuanto al factor de la dilatación en relación con la asistencia? ¿Qué requisitos necesitaría especialmente?
Doctor Báez: - Los requisitos dependen en primer lugar de la paridad, en segundo lugar de la distancia a que ella vive del hospital en donde va a tener su parto. Eso se tiene en cuenta para que el tiempo para trasladarse a la maternidad en relación con la frecuencia de las contracciones, entonces no se cuida como la primípara, en quien las contracciones no son cada diez minutos o menos sino cada quince minutos.

¿Existe un problema en esto de que ingresen con el trabajo de parto adelantado? NINGUNO. Aunque pudiese pensarse que fuera así, sobre todo en lo que respecta al sufrimiento fetal, que podría instalarse con bolsa íntegra, porque no ha perdido agua, que es otro motivo de internación, y pasar entonces desapercibido para la señora; pero en la práctica no hemos tenido problemas.

3. Al doctor Silva: - iEl celo del obstetra en mejorar la calificación de la paciente puede tener influencia nociva sobre el resultado final?

Igualmente, el miedo de la paciente de hacer quedar mal a su médico, ¿puede interferir nocivamente en ésta? 
Doctor López-Escobar: -Creo que se debe evitar el hablar a la paciente sobre calificaciones.

Doctor Silva: - Bueno, creo que es favorable el interés del obstetra por obtener buenos resultados, ya que en estos métodos existe un factor sugestivo; naturalmente que si el médico no está convencido de obtener buenos resultados, seguramente va a fracasar. Es posible que dado el factor sicológico que han visto en algunas enfermas del temor a fracasar y hacer quedar mal a su médico, influya en la paciente.

Doctor Bussamara: -Nosotros pensamos que todo médico con algún ascendiente sobre su paciente, que le dice a la enferma que le va a dar una nota por su comportamiento durante el parto, a veces se preocupan más en satisfacer a su médico que por ellas mismas, tanto así que cuando se hace anestesia al final, especialmente con barbitúricos, la paciente siempre reacciona pidiendo disculpas al médico y llorando cuando se da cuenta de que la tuvieron que anestesiar, porque siente que lo ha defraudado. Hay inclusive algunas mujeres que tratan de rechazar la anestesia para hacerse agradables a su médico.

Doctor Báez: -Sistemáticamente nosotros en la preparación, le decimos a la paciente, en la primera clase, que el resultado depende fundamentalmente de ella, para que de esa manera pueda crear focos realmente positivos en ella. No somos partidarios de recoger encuestas sobre cómo se desarrolló el parto, porque allí tiene toda la in- fluencia síquica de lo que acaba de citar el doctor Neme Bussamara. Y en los casos en que hubiera fracasado, nosotros creamos un optimismo en ella, sin relevar nunca que el tratamiento no dio todos los resultados que eran de esperar, con el objeto de que no se creen focos fuertemente negativos en ella para un parto posterior, sino al contrario.

Doctor Navas: -Estamos perfectamente de acuerdo con lo que dice el doctor Báez; se ha insistido tanto en las estadísticas que se ha creado una sicosis de estadísticas, hasta tal punto que cada cual quiere presentar resultados sobre bases tan heterogéneas, que no se pueden establecer estadísticas al respecto. Pero sí es absolutamente cierto que las señoras se esfuerzan estoicamente en no hacer quedar mal a su médico, disimulando el dolor y la ansiedad para decir que triunfó. Es necesario decirles, como lo hacemos nosotios, que el resultado no tiene relación ninguna con el éxito profesional. No hemos descubierto nosotros el método, y el decírselo es suficiente.

4. Para el doctor Arenas: - ¿Por qué razón no se tiene en cuenta la aplicación de Demerol en la evaluación de los resultados en el método sico-profiláctico, siendo así que esta droga disminuye hasta un cincuenta por ciento la percepción del dolor?

Doctor Arenas: -En cuanto a la aplicación del Demerol, tenemos dos criterios: Si la paciente está en un descontrol emocional y acusando dolor que puede llegar a ser intolerable, se aplica 
el Demerol y la estabilización baja indudablemente a regular, se aplicó para disminuír el dolor y estabilizar emocionalmente a la paciente. En cambio, si la paciente en perfecto equilibrio emocional, en que no acusa dolor ninguno, que tenga una polisistolia, entonces usamos el Demerol para coordinar el trabajo y en este caso no se baja la calificación, ya que la finalidad no fue estabilizada emocionalmente, ni calmar el dolor, sino coordinar el trabajo.

Doctor Pérez Sánchez: -Nosotros hemos usado el Demerol a larga mano, tanto en las pacientes preparadas, como en las no preparadas; y podemos decir que en una madre preparada sico-profilácticamente, es muy corriente el observar su desacondicionamiento. Hemos tratado de explicarnos este efecto y parecería ser que el Demerol baja el umbral al dolor al mismo tiempo que libera la conciencia de la madre, que, dada su preparación, la ha tenido restringida, controlada en sus reacciones: la madre se alivia, se le arregla su polisistolia, se nos desacondiciona.

Doctor Arenas: - En parte estoy de acuerdo con el doctor Pérez Sánchez, pero he creído que eso depende de la dosis de Demerol y de la vía que se use. Nosotros usamos $50 \mathrm{mgrs}$. por vía intramuscular.

Doctor Pérez Sánchez: - Habitualmente usamos 50 mgrs. de Demerol, por vía endovenosa.

Doctor Navas: - Sobre la aplicación de Demerol, lo acaban de decir, depende de dosis. No puede intentarse nun- ca, porque es absolutamente imposible, quitar los dolores del parto con Demerol; simplemente se trata, con algunos coadyuvantes, para coordinar un trabajo de parto, también es un medio de hipnosis decirle a una paciente, que está descontrolada, que con la inyección que se le va a poner se va a regularizar su contracción, y aun cuando algunos afirman que la dosis de 50 mgrs. es inoperante, muchas veces la contracción se regulariza y el parto sigue su curso normal después.

Doctor Pedro García: -Nosotros usamos el Demerol por excepción, únicamente en aquellas señoras en que por se temperamento excitadas, necesitamos disminuír el funcionamiento de su corteza cerebral, para que de esta manera se puedan equilibrar; de lo contrario siempre usamos cuando se hace necesario un medicamento, utilizamos Meprobamatos.

Doctor Bussamara: -Nosotros pensamos en relación con el Demerol y demás analgésicos, lo siguiente: La respuesta es individual en todas las pacientes que reciben el sedativo y están condicionadas, dependiendo del grado de intensidad de acción central de esas drogas y del aspecto de condicionamiento de aquellas en el parto. De modo que puede ser inyectada y lograr el efecto hipnótico de que habla el doctor Navas. Ahora bien, nosotros no pensamos que la acción de la droga sea únicamente síquica, la verdad es que aun si la paciente no siente nada, mejora la contracción.

Doctor García: -Nos parece que el uso del método sico-profiláctico no 
contraindica ningún otro medio obstétrico con el cual se pueda mejorar la dinamia, o aun el estado sicológico de la mujer. Se aconseja, sí, me parece que es general la opinión de todos los que emplean este método, que se use la menor cantidad de drogas posible, pero sin contraindicar ninguna. Habría que tener en cuenta sólo aquellas que inhiben la corteza cerebral, puesto que nosotros tratamos de conseguir el máximum de conciencia y la mayor actividad cortical posible. El Demerol parece que tendría una cierta actividad inhibitoria sobre la corteza cerebral. Es por eso por lo que cuando debamos emplearlo, se hará en la dosis mínima posible; nunca los 100 mgrs. de la ampolla completa, 50 o aun 25 mgrs. podrían bastar.

5. Al doctor Báez y al doctor Arenas: - ¿Qué método de inducción hipnótica emplean en los casos preparados y en los extemporáneos?

Doctor Báez: -Nosotros hacemos una inducción hipnótica con la prueba de susceptibilidad, pero esto es en casos excepcionales; no es en la práctica en grandes números, la parte hipnosis es muy limitada. Nosotros seguimos el sistema de Duplatt, que es una forma de seguir en la Argentina, de acuerdo con Duplatt.

Doctor Arenas: -En los casos preparados hacemos una hipnosis colectiva: por relajación y sin hablar de hipnosis. Cuando se hace hipnosis sin hablar de ella, hay que justificar cada sugerencia, es prácticamente un engaño, una mentira piadosa. Si ella acepta, co- mo generalmente sucede, entonces está manejando la idea que le sugerimos y por lo tanto resulta la hipnosis.

En la preparación de extemporáneos seguimos un método diferente; les aclaro que también seguimos al profesor Duplatt, quien estuvo con nosotros en Bucaramanga varias semanas. En la preparación extemporánea generalmente encontramos una paciente llorando, gritando y quejándose a cada contracción; le decimos que nada pierde con respirar en esta forma y tratar de aflojarse. Ella probablemente no cree, pero si acepta la sugerencia y la practica en la siguiente contracción, y le decimos que en la otra contracción si vuelve a hacer lo mismo, le va a doler aún menos, y acepta, le duele menos, ya que toda idea tiene su correlativo fisiológico. En la tercera contracción le instalamos nuevamente la misma sugerencia, pero diciéndole que en un alto porcentaje ya no le duele casi nada o nada. Entonces seguramente resulta en un alto porcentaje. Luego le decimos que se ponga tensa y no respire y verá cómo le duele tres veces más, que con seguridad ella lo va a sentir así; aquí estamos instalando una sugerencia de hiperestesia. Esa paciente no dejará en lo sucesivo de respirar y mantenerse relajada, y por lo menos si le duele mantendrá una conducta apropiada.

6. A quién la quiera contestar: - ¿Da mejores resultados el método sico-hipnótico haciéndolo individualmente que colectivamente?

Doctor Fernández: - Creo que individualmente el rapport se hace mejor, 
la paciente puede manifestar sus inquietudes. El método colectivo tendría una desventaja a mi modo de ver.

Doctor Báez: -Estoy totalmente de acuerdo con lo que acaba de decir el doctor Fernández, pero encuentro que justamente en la que sigue, en la preparación sico-profiláctica se puede utilizar el método colectivo.

Doctor Navas: - Creo que en esta preparación no se debe reunir ni un gran número de pacientes, lo que las cohibe unas a otras, ni tampoco hacerse individual. La preparación ideal es de grupos de seis a ocho. Individualmente puede existir también inhibición de la paciente con su médico, que no le tiene la suficiente confianza, etc.

Doctor García: - Justamente podríamos pensar que la ventaja del método sico-profiláctico permite preparar un gran número de personas, sería nuestro ideal que todas las mujeres que concurren a un servicio pudieran seguir esta preparación. Fácilmente se comprende que esto es imposible hacerlo individualmente. De allí que en Rusia se pasaran del método hipnótico, que no se podía aplicar a las masas, al método sico-profiláctico, que seguimos actualmente, porque es un método de masas. Lo que acaba de decir mi antecesor es perfectamente válido. Esto tiene que hacerse en pequeños grupos, máximum debería ser de unas doce. Allí hay una serie de factores que intervienen favorablemente: casi todas han tenido algún antecedente de una conocida que ha seguido este método y es por eso por lo que ellas procuran ir; éstas in- fluyen favorablemente en todas las demás, se hacen comentarios mutuos entre ellas sobre lo que ya conocen de los éxitos anteriores, de lo que van aprendiendo sucesivamente, de cómo les interesa lo que se les dice, y de cómo va cambiando gradualmente el ambiente colectivo, no sólo en ese grupo sino en la familia misma. Es sumamente importante en la preparación de la embarazada la presencia y preparación simultánea del marido, que va a ser el punto de apoyo en el hogar, para que la señora siga preparándose en su casa, cosa que no hará si no tiene el control de su marido, le falta el apoyo necesario.

7. Del doctor Bussamara al doctor Silva Mojica: -Pensamos que la presencia del médico, especialmente durante el período de transición entre la dilatación y la expulsión favorecería mucho los resultados finales. ¿Qué opina usted de esto?

Doctor Silva: -Ese es un momento, según lo comprobamos con diversos estudios al respecto, durante el cual las mujeres se desacondicionan más. De manera que consideramos absolutamente indispensable la presencia del obstetra en ese momento fuera de la de una instructora, que está desde el comienzo del trabajo. Debe ser así y así lo hacemos.

Doctor Pérez Sánchez:-Estoy perfectamente de acuerdo con lo que han dicho; hasta tal punto es necesaria la presencia del obstetra en ese período de transición, que en los fracasos obstétricos que hemos presentado antes, 
culpamos de esta falta de éxito a la ausencia del obstetra en dicho momento; sin embargo, creemos que, como lo dijo el doctor García, este sistema sico-profiláctico debe de ser orientado en un sentido de masas, eliminando de la mente del médico y de la paciente el que sea un sistema reservado a la élite. Realmente no veo cómo podría ensamblarse un sistema en ei cual el médico que prepare un gran número de madres pudiera estar presente en el período de transición de cada madre.

Doctor García: -Precisamente esto debe ser una labor de equipo; es necesario que la paciente no crea indispensable una presencia determinada con ella o la de todo el equipo, teniendo confianza en éste, de modo que si por una circunstancia fortuita uno de los miembros faltare, la parturienta tenga la plena seguridad de que cualquier otro va a desempeñar la misma labor con el mismo éxito. Ha de tratar de aminorarse la influencia, innegablemente muy grande, del médico o preparador.

Doctor Pérez Sánchez: - $\mathrm{Si}$ existe espíritu de equipo, no hay ninguna dificultad para que el éxito sea rotundo si falta el preparador.

Doctor Fernández: -Estoy naturalmente de acuerdo en que la presencia del médico tenga una influencia inmensa sobre la paciente cuya preparación él mismo ha hecho antes. Ahora, este método debe generalizarse, como dice el doctor Pérez Sánchez; allá llegaremos, pero este es un método muy nue- vo; así es que tenemos que empezar por eliminar en todas las mujeres esas ideas anticuadas del parto doloroso. La generalización vendrá paulatinamente, hoy es indispensable la presencia del médico en el período transitorio de dilatación a expulsión, ya que si fracasa con lo primero que vamos a hacer, ¿quién lo va a seguir después? Además, nuestros medios de preparación del pueblo son tan malos en el alfabeto, con mayor razón en estos otros métodos. Tenemos que comenzar a educar al pueblo al respecto ahora para años posteriores.

8. Al doctor Silva Mojica: - En caso de encontrar problemas de índole sicológica, ¿qué modificaciones se imponen en la preparación y atención del parto de dichas pacientes?

Doctor Silva Mojica: - En el trabajo tratamos de precisarlo, siendo entre nosotros bastante frecuentes los problemas de índole sicológica. Los principales son: Rechazo al embarazo, Inmadurez sico-sexual, Complejo de inferioridad y Problemas de sobreprotección. Por el estudio sicológico que hicimos, llegamos a la conclusión de que se necesita una sico-terapia especial. Verlaine nos lo había recomendado ya. En vista de la alta frecuencia de este género de problemas entre nosotros se hace indispensable una mayor colaboración del siquiatra; de ahora en adelante nos empeñaremos en que así sea, ya que alrededor del noventa por ciento de las pacientes necesitan un tipo de sico-terapia especial que requiere la colaboración del siquiatra durante la preparación. 
Doctor Báez: —Esto pone en evidencia las diferencias existentes entre los diversos medios, nosotros tenemos un porcentaje muy bajo de casos en los que no se puede prescindir de la consulta con el siquiatra. Consideramos al siquiatra como un elemento consultor más, como ocurre en otros órdenes de la práctica obstétrica.

9. A la Mesa: -Experiencia de la Mesa sobre la influencia de los tranquilizantes, administrados durante el embarazo y causantes de malformaciones fetales o de taras congénitas.

Doctor Báez: -No utilizamos nunca los tranquilizantes al comienzo del embarazo; creemos que el tratamiento de hipermnesia gravídica es fundamentalmente de orden sico-terápico. La experiencia que tenemos con tranquilizantes es especialmente en el último mes del embarazo y desde luego no obtenemos ninguna clase de malformaciones.

Doctor Silva Mojica: -No tengo ninguna experiencia en este campo.

Doctor Bussamara: -En clínica privada damos, si es necesario, Meprobamatos, nunca tranquilizantes. Nunca observé malformaciones como consecuencia de la administración de Meprobamatos, que en el Brasil se usan bastante.

Doctor Navas: - Creo que se teme mucho a los tranquilizantes; nosotros los usamos relativamente poco durante el embarazo. Estamos más de acuerdo con la hipnosis en casos graves de vómitos incoercibles, que son relativa- mente escasos ahora. No ha habido ninguna malformación por el uso de los tranquilizantes.

10. - ¿Cree la Mesa que la preparación sico-profiláctica y la hipnosis deban ser usadas conjuntamente, o por el contrario se deben mantener separados los miembros de uno y otro grupo, a fin de evaluar resultados?

Doctor Arenas: -En Bucaramanga creemos que la hipnosis debe usarse como un refuerzo a la preparación sicoprofiláctica porque el que está haciendo una preparación de este tipo propone a sus pacientes y éstas están aceptando, siendo mejores los resultados en los grupos preparados por un médico con bastantes capacidades sugestivas. Entonces como el factor sugestivo no se puede despreciar en la preparación sico-profiláctica, hemos reforzado esta preparación con hipnosis.

11. Al doctor Pérez Sánchez: --¿Cuáles son los motivos de las causas del fracaso de acuerdo con los resultados obtenidos en la Universidad Católica de Chile?

Doctor Báez: -En primer lugar yo no hablaría de fracaso, porque, como lo dije al principio de la exposición, tuvimos gran éxito en el acondicionamiento de la madre y el alivio del dolor. No traje porcentajes ni evaluaciones, como las otras que vimos, porque no era el objeto de la presentación. Sin embargo, en lo obstétrico puro no obtuvimos éxito. Me gustaría ver trabajos en que me mostraran éxitos rotundos en lo obstétrico para poder entonces hablar de fracaso en nuestra ope- 
ración. Como evidentemente no obtuvimos lo que buscábamos, que era acortar el parto, disminuír el expulsivo, disminuír la distocia, pensamos que las causas son las siguientes: Nosotros trabajamos especialmente con población económico-social baja, tratamos de darle un sistema práctico, que hiciera posible concurrir a la madre a las clases sacándola poco de su casa; así hicimos sólo tres clases teóricas y dos reuniones prácticas. Eso y la falta de uno de los miembros del equipo preparador, que a veces no estaban en el momento del parto, podrían haber sido las causas de los desacondicionamientos de las madres.

12. Para el doctor Pérez Sánchez: -Por estas experiencias que presentan aquí en el Congreso, ¿abandonaron en Chile la preparación sico-profiláctica para el parto?

Doctor Pérez Sánchez: - No, en absoluto. Esta revisión que trajimos nos sirve para modificar un poco nuestra forma de trabajo, haciendo más extensivo el conocimiento a todo miembro del personal, pero en ningún modo nos sentimos desilusionados de esto, seguiremos siendo uno de los equipos pioneros de la sico-profilaxis en la Universidad Católica de Chile, lo digo humildemente.

Doctor García: - Creo que aquí hay una equivocación de términos; el doctor Pérez ha dicho que no obtuvo mejoría en la parte obstétrica, pero tengo entendido que los éxitos que se lograron en cuanto al método en sí, son los mismos que se alcanzaron en todas partes, y que se han mostrado aquí.
Rev. Col. Obst. y Ginec.

Ahora, las causas de los fracasos son las mismas que aquí se han enumerado. Ahora las otras causas, especialmente la hiperdinamia p. e., son cosas que no dependen del método en sí mismo.

Además es necesario que todos sepan y lo adviertan a las mujeres, que no hay en medicina ningún método que dé siempre un éxito ciento por ciento.

Doctor Bussamara: -Existe un trabajo de una clínica de Ginebra, bastante perfecto, que compara el resultado obstétrico de todas mis pacientes indistintamente escogidas, en las que no se había practicado preparación sicológica y otras en las que sí. Ellos no encontraron modificaciones dignas de anotar en relación con el parto, especialmente en cuanto al número y frecuencia de las intervenciones, ya sea por vía alta o baja. Ahora, es necesario admitir conjuntamente los efectos de la preparación sico-profiláctica, con sus desventajas o incidentes. La pregunta que acaba de ser hecha es muy difícil de contestar, ya que no se puede hacer una evaluación precisa de los resultados obtenidos con este método.

Al doctor Pérez Sánchez: - $-i$ Con qué fin practican en Chile el método sico-profiláctico?

Doctor P. Sánchez: -Para obtener un alivio del dolor en el parto. Sin embargo, en toda publicación de sicoprofilaxis se hace referencia a los tiempos obstétricos, a la distocia, a la dinamia y al número de intervenciones. El objetivo, como pregunta el doctor Navas, es el alivio del dolor en el parto. 
Doctor García: -Indudablemente queremos obtener la mayor parte de indolorización en el parto, pero con ser esto un fin principal, no es el único. Pretendemos hacer la educación del medio femenino, higiene mental, deshacer lo que miles de años de tradiciones absurdas han hecho, con un uso razonado de la palabra.

13. A la mesa: - Internacionalmente, ¿cuál es la calificación aceptable sobre las pacientes atendidas con el método sico-profiláctico?

Doctor Báez: - Creo que desde hace mucho tiempo estamos en una etapa de preparación dentro de la sicoprofilaxis. Los resultados finales son los que valen. Hasta ahora me ha tenido sin cuidado esta calificación.

Doctor Silva Mojica: —La clasificación de las calificaciones existe y es la que mencioné en el trabajo, la de la Escuela de Lamas, ellos la siguen. Ahora existen otras clasificaciones, pero esta clase de cosas siempre tiene sus problemas. Inclusive algunos adictos a Lamas, aquí presentes, estuvieron de acuerdo con nosotros en que se debería simplificar un poco, y de ahí a que evolucionara un poco esta clasificación colombiana. Es difícil por esto la comparación de estadísticas. De todas maneras quiero recordarles que actualmente es imposible, dentro del método sico-profiláctico, presentar resultados absolutos, ni definitivos. 